\title{
Volume of Distribution of Absorbed
}

\section{Fraction}

National Cancer Institute

\section{Source}

National Cancer Institute. Volume of Distribution of Absorbed Fraction. NCI Thesaurus.

Code C85541.

The volume of distribution of the absorbed fraction, where F equals the fraction of dose absorbed. 